\title{
FAST HIGH-RESOLUTION PREDICTION OF MULTI-PHASE FLOW IN FRACTURED FORMATIONS
}

George Shu Heng Pau ${ }^{1}$

gpau@lbl.gov (Corresponding author)

Stefan Finsterle ${ }^{2}$

safinsterle@lbl.gov

Yingqi Zhang ${ }^{2}$

yqzhang@lbl.gov

${ }^{1}$ Lawrence Berkeley National Laboratory, Climate and Ecosystem Sciences Division, 1 Cycloton Road, Berkeley, California

${ }^{2}$ Lawrence Berkeley National Laboratory, Energy Geosciences Division, 1 Cycloton Road, Berkeley, California

\section{$1 \quad$ ABSTRACT}

2 The success of a thermal water flood for enhanced oil recovery (EOR) depends on a detailed

3 representation of the geometrical and hydraulic properties of the fracture network, which induces discrete,

4 channelized flow behavior. The resulting high-resolution model is typically computationally very

5 demanding. Here, we use the Proper Orthogonal Decomposition Mapping Method to reconstruct high-

6 resolution solutions based on efficient low-resolution solutions. The method requires training a reduced

7 order model (ROM) using high- and low-resolution solutions determined for a relatively short simulation

8 time. For a cyclic EOR operation, the oil production rate and the heterogeneous structure of the oil

9 saturation are accurately reproduced even after 105 cycles, reducing the computational cost by at least

$1085 \%$. The method described is general and can be potentially utilized with any multiphase flow model. 
13 Efficient numerical approaches are needed to lower the computational barrier for performing optimization

14 and uncertainty quantification using models that accurately represent complex multi-phase flow processes

15 in fracture networks, with considerable impact on our ability to sustainably manage and optimize energy

16 and water resource systems, and to effectively remediate contaminated sites. For example, a reliable

17 evaluation of the economic viability of thermal water flood-a common enhanced oil recovery (EOR)

18 technique - depends on whether we can predict the oil production rate accurately. Prediction of the oil

19 production rate is typically obtained by constructing a numerical model that accurately captures the

20 geometrical and hydraulic details of the fracture network, which induces discrete, channelized flow

21 behavior. The network also determines the effectiveness with which heat and brine penetrates the rock

22 matrix, mobilizing and displacing the oil. Simulating an EOR operation using a discrete fracture network

23 embedded in a low-permeability matrix is computationally very demanding, mainly because the detailed

24 representation of the fracture network and the complex geometry of the matrix blocks bounded by

25 randomly oriented fracture planes requires high-resolution meshes.

27 In this paper, we apply a reduced order modeling (ROM) technique known as the Proper Orthogonal

28 Decomposition Mapping Method (PODMM) — first proposed by Robinson et al. [1] — which allows us to

29 reconstruct the solutions from a high-resolution model representing the fracture network as a

30 heterogeneous medium based on solutions obtained using low-resolution models that only have upscaled,

31 effective properties of the fracture network and thus can be efficiently simulated. This technique was

32 recently enhanced and applied to land surface models to accurately reconstruct hydrological states, heat

33 fluxes, and carbon fluxes [2, 3]. However, the suitability of the method for modeling multiphase non-

34 isothermal subsurface problems with significant nonlinear temporal and spatial dynamics has yet to be

35 demonstrated. This work evaluates the accuracy of PODMM for a multiphase problem (an enhanced oil 36 recovery problem). 
PODMM can be viewed as a regression-based downscaling technique. Overviews of empirical downscaling techniques have been presented before (see, e.g., Wilby et al. [4], Fowler et al. [5], Gutmann et al. [6]). Previous work on regression-based downscaling methods focuses on constructing empirical parametric models between the predictors and variables of interest $[7,8]$. In the context of reduced order models (ROMs), regression models can also be constructed between model parameters and the variables of interest $[9,10]$. PODMM differs from the above regression approaches in that proper orthogonal decomposition (POD) is not just used to obtain a dimensionally reduced representation of the highdimensional data. Instead, a least-square minimization problem that utilizes portions of the singular vectors obtained through the POD procedure [11] is solved to directly map low-resolution solutions to the high-resolution solutions. More details are provided in Section 1.2.

PODMM also differs from the projection-based POD methods that were previously applied to subsurface flow problems [12-15] and other engineering fields [16-18]. Specifically, the projection-based POD method is an intrusive approach that requires projecting the governing partial differential equations onto a linear space spanned by the singular vectors, and implementing the resulting discrete equations. For a multiphase non-isothermal model, the complicated nonlinear terms require additional approximations [19simpler since it only requires solving the low-resolution models once the ROMs have been trained. Thus,

56 it does not require intrusive changes to the simulation software, making it an attractive method for 57 complex multiphase flow problems.

\section{METHODS}

\subsection{Mapped Fracture Network Models}

60 We demonstrate the proposed PODMM model-reduction approach for an EOR operation conducted in a

61 fractured hydrocarbon reservoir with a single injection-extraction well. An individual cycle of the 62 operation consists of four phases: (1) injection of hot water at $10 \mathrm{~kg} / \mathrm{s}$ for 3 days, (2) an inactive soaking 
period of 4 days, (3) production of oil and water for 6 days at a total rate of $5 \mathrm{~kg} / \mathrm{s}$, and (4) an inactive rest

64 period of 1 day. This two-week cycle is repeated 105 times for a total simulation time of 1470 days. The distribution of oil in the reservoir and the oil production rates are the key prediction variables of interest.

67 We consider a discrete fracture network within a model domain of dimensions $100 \times 50 \times 30 \mathrm{~m}$. Fractures are generated by randomly sampled values for size, orientation, and aperture from appropriate, truncated probability distributions. Two fracture sets with an average fracture spacing of $4 \mathrm{~m}$ are generated using the code ThreeDFracMap [22]. In our modeling approach, the fracture network is then represented by a heterogeneous continuum model. The fractures are first mapped onto a structured, uniform mesh, before upscaled, heterogeneous, anisotropic permeabilities are calculated based on the number, aperture, and orientation of the fractures intersecting the given element. Elements that do not contain any fractures are assigned a low matrix permeability of $10^{-18} \mathrm{~m}^{2}$. The procedure is described in detail in Parashar and Reeves (2011).

Based on this heterogeneous, high-resolution continuum model representing a network of discrete features embedded into a low-permeability matrix, we use the "dead-oil" (EOS8) module of TOUGH2 [23] to simulate the response of the reservoir to cyclic injection of hot water and production of a multiphase mixture of oil and water. Simulating a long sequence of injection-production cycles is computationally expensive, especially if a high-resolution continuum representation of the discrete network is needed to capture the exchange of fluids between the reservoir rock (which contains most of the oil) and a network of discrete fractures (whose main role is to provide the pathways for oil extraction)

84 embedded in that matrix. Moreover, the resolution also affects the system behavior and computational 85 costs. The PODMM approach described below predicts the high-resolution behavior using a computationally efficient low-resolution model (LRM), combined with a mapping procedure for

87 downscaling the simulation results. Two basic grids with different resolutions are thus developed: a high- 
size of $5 \mathrm{~m}$ (see Figure 1(a)). The LRM thus has about 15 times fewer elements than the HRM, making it

90 significantly more efficient at the expense of loss of accuracy in representing discrete flow behavior in the

91 fractures and fluid exchange with the matrix. The HRM provides simulation data for a relatively short

92 training phase; it is also used in this study as the reference solution needed to demonstrate the accuracy of

93 the proposed approach. The LRM provides approximate, efficient solutions for the entire simulation

94 period; these solutions are then downscaled to provide high-resolution predictions of the cyclic EOR

95 operation based on the mapping procedure in PODMM.

96

97 We examine three alternative LRMs: (1) an upscaled heterogeneous model (LRM-HET), using the exact

98 same conceptualization as the HRM with the exception that it uses a coarser discretization, (2) a simple

99 homogeneous model (LRM-HOM) with a permeability of $10^{-13} \mathrm{~m}^{2}$, and (3) a dual-porosity model (LRM-

100 DPM) [24] with fracture- and matrix-continuum permeabilities of $10^{-13} \mathrm{~m}^{2}$ and $10^{-18} \mathrm{~m}^{2}$, respectively. Key

101 parameters are summarized in Table 1. Note that the number of elements of LRM-DPM is twice that of

102 LRM-HOM and LRM-HET. The permeabilities of LRM-HOM and LRM-DPM are chosen to

103 approximately represent the fracture network permeability such that they produce some of the behaviors

104 seen in the HRM. However, no rigorous upscaling technique is used to determine the permeabilities of the

105 LRMs, e.g., by matching the oil production rate obtained from the HRM through an inverse modeling

106 procedure. A calibrated LRM will likely improve the performance of the PODMM. However, the goal of

107 this work is to demonstrate that the accuracy of the PODMM is not due to the calibration of LRM model

108 parameters to fit the outputs of the HRM.

109

110 Figure 1(b) shows the oil saturation after 3 days. The solutions obtained from LRM-HET is a smoother

111 representation of the considerably more intricate distribution obtained with the HRM. Figure 1(c), and

112 Figure $1(\mathrm{~d})$ compare oil production rate $\left(Q_{\mathrm{oil}}\right)$ determined using the HRM and the $3 \mathrm{LRMs}$ at the $55^{\text {th }}$, and

$113105^{\text {th }}$ cycles, respectively. For the parameters given in Table 1, LRM-HET over-predicts the $Q_{\text {oil }}$ while

114 LRM-HOM and LRM-DPM under-predict the $Q_{\text {oil }}$. 
116 Table 1. Summary of key geometric, hydraulic, and thermal model parameters

\begin{tabular}{|l|c|c|c|c|}
\hline \multirow{2}{*}{ Parameter } & \multirow{2}{*}{ HRM } & \multicolumn{3}{|c|}{ LRM } \\
\cline { 3 - 5 } & & LRM-HOM & LRM-DPM & LRM-HET \\
\hline Domain size, $\mathrm{m}$ & \multicolumn{4}{|c|}{$100 \times 50 \times 30$} \\
\hline Element size, $\mathrm{m}$ & 2 & 5 & 5 & 5 \\
\hline Number of elements & 18,750 & 1,200 & 2,400 & 1,200 \\
\hline Matrix permeability, $\mathrm{m}^{-18}$ & $10^{-18}$ & $\mathrm{~N} / \mathrm{A}$ & $10^{-18}$ & $10^{-18}$ \\
\hline $\begin{array}{l}\text { Mapped fracture network } \\
\text { permeability, } \mathrm{m}^{2}\end{array}$ & $\begin{array}{c}\text { heterogeneous, } \\
\text { anisotropic }\end{array}$ & $\begin{array}{c}10^{-13} \\
\text { isotropic }\end{array}$ & $\begin{array}{c}10^{-13} \\
\text { isotropic }\end{array}$ & $\begin{array}{c}\text { heterogeneous, } \\
\text { anisotropic }\end{array}$ \\
\hline porosity, \% & 4 & 4 & 4 & 4 \\
\hline heat conductivity, $\mathrm{W} \mathrm{m}^{-1} \mathrm{~K}^{-1}$ & 2.5 & 2.5 & 2.5 & 2.5 \\
\hline heat capacity, $\mathrm{J} \mathrm{kg}^{-1} \mathrm{~K}^{-1}$ & 1000 & 1000 & 1000 & 1000 \\
\hline pore compressibility, $\mathrm{Pa}^{-1}$ & $10^{-9}$ & $10^{-9}$ & $10^{-9}$ & $10^{-9}$ \\
\hline
\end{tabular}

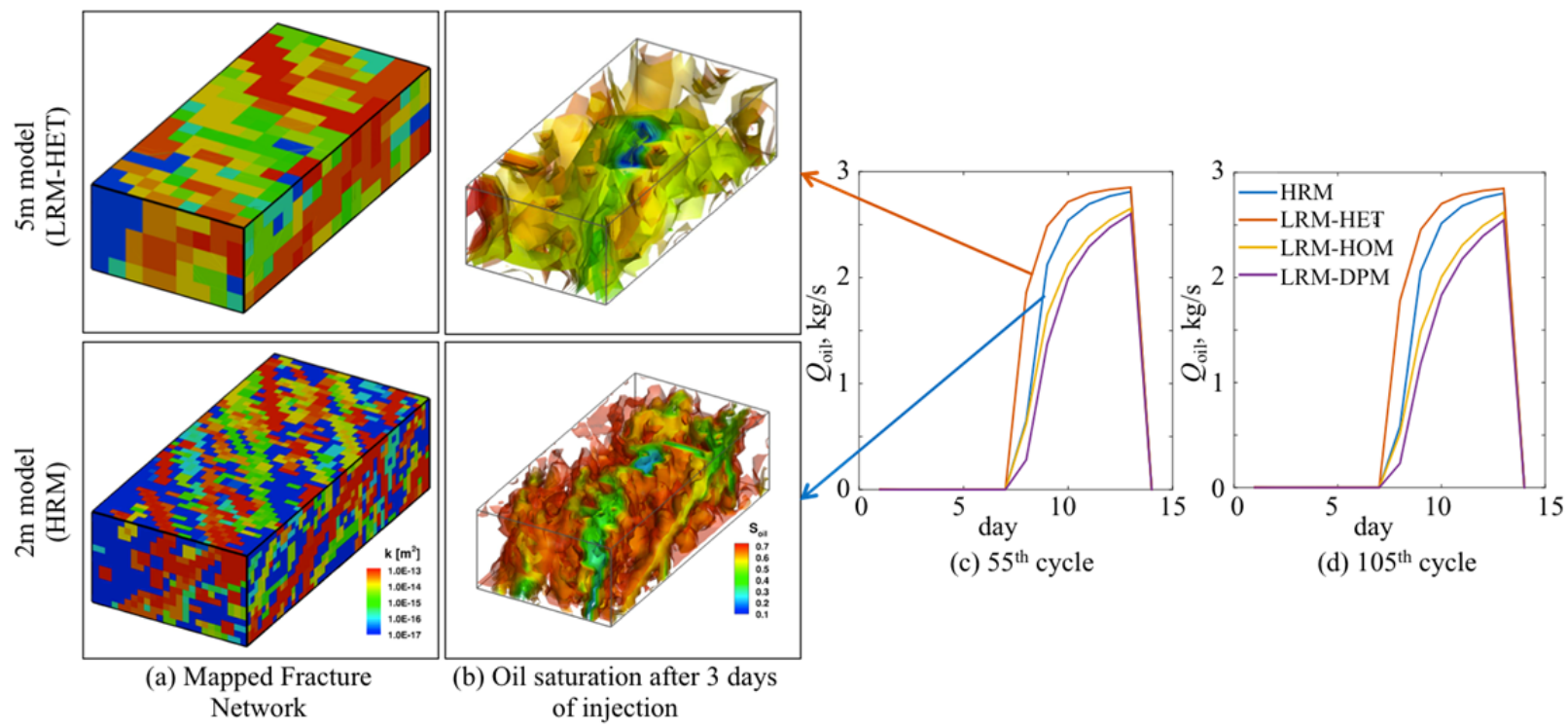

119 Figure 1. Discrete fracture network mapped onto TOUGH2 continuum grid using (a) a low resolution of 5 m (LRM-HET) (top), and a high resolution of $2 \mathrm{~m}$ (HRM) (bottom). Oil saturation after 3 days of hot water injection, calculated with (b) LRM-HET (top), and HRM (bottom). The comparison of oil production rate $\left(Q_{\text {oil }}\right)$ curves at the (c) $55^{\text {th }}$ cycle, and (d) $105^{\text {th }}$ cycle for the HRM and the LRMs.

\subsection{Proper Orthogonal Decomposition Mapping Method}

126 found in Pau et al. [2]. The method consists of a training stage and a prediction stage. During the training 
127 stage, we determine the solutions (e.g., oil saturations and fluxes at all locations) to the low-, and high128 resolution models (denoted as $\mathbf{g}$, and $\mathbf{f}$, respectively) at $N$ time points. These $N$ solutions constitute the 129 training set. In our example, snapshots are obtained at 1-day intervals from multiple consecutive EOR 130 cycles. We then perform a singular value decomposition (SVD) of the following matrix $\mathbf{W}$ :

$$
\mathbf{W}=\left[\begin{array}{lll}
\mathbf{f}_{1}-\overline{\mathbf{f}} & \mathbf{f}_{N}-\overline{\mathbf{f}} \\
\mathbf{g}_{1}-\overline{\mathbf{g}} & \cdots & \mathbf{g}_{N}-\overline{\mathbf{g}}
\end{array}\right]
$$

132 where $\mathbf{f}_{i}$, and $\mathbf{g}_{i}$ are the high-, and low-resolution solutions at the $i^{\text {th }}$ time point,

$$
\overline{\mathbf{f}}=\frac{1}{N} \sum_{i=1}^{N} \mathbf{f}_{i}, \quad \overline{\mathbf{g}}=\frac{1}{N} \sum_{i=1}^{N} \mathbf{g}_{i}
$$

134 The POD bases, $\zeta_{i}, i=1, \ldots M$, are given by the resulting singular vectors and can be decomposed into

$$
\zeta_{i}=\left[\begin{array}{c}
\zeta_{i}^{\mathbf{f}} \\
\zeta_{i}^{\mathbf{g}}
\end{array}\right]
$$

where $\zeta_{i}^{\mathbf{f}}$ and $\zeta_{i}^{\mathbf{g}}$ are components associated with the HRM and LRMs, respectively, and $M$ is the chosen

137 number of POD bases to use in an approximation.

142 downscaled high-resolution solution $\mathbf{f}^{\mathrm{ROM}}$ is then given by $\overline{\mathbf{f}}+\sum_{i=1}^{M} \alpha_{i}(\mathbf{g}) \zeta_{i}^{\mathbf{f}}$.

144 We can jointly consider multiple variables in a single ROM to take advantage of correlations between the 145 variables by modifying matrix $\mathbf{W}$ in Eqn. (1) such that each column consists of concatenated solutions of 146 these variables (from both low- and high-resolution models). See [3] for a more complete description of 
147 the method. For the current study, we jointly predict all primary variables of the model, i.e., the oil 148 saturation $\left(\boldsymbol{S}_{\text {oil }}\right)$, pressure $(\boldsymbol{P})$ and temperature $(\boldsymbol{T}) ; \mathbf{g}$, and $\mathbf{f}$ in Eqn. (1) are then given by $\left[\boldsymbol{S}_{\text {oil }} ; \boldsymbol{P} ; \boldsymbol{T}\right]$ of the 149 low-, and high-resolution models, respectively. Since these variables have disparate magnitudes, they are 150 first rescaled by their means. Similarly, we create a ROM for the fluxes by jointly predicting the flux 151 distributions of oil $\left(\boldsymbol{F}_{\text {oil }}\right)$, water $\left(\boldsymbol{F}_{\text {water }}\right)$ and heat $\left(\boldsymbol{F}_{\text {heat }}\right)$ over the entire domain.

153 The main model parameter for the ROM is the parameter $M$, the number of bases to use in the 154 approximation. It controls the accuracy and stability of the approximation. A common heuristic approach 155 to determining an appropriate $M$, denoted by $M_{R}$, is by specifying a desired percentage of variance 156 explained, obtained by summing the normalized singular values associated with the $M_{R}$ selected POD 157 bases $[10,15]$. However, this approach favors the use of large $M_{R}$, which can be unsuitable in a 158 PODMM approximation since it can lead to overfitting when solving the minimization problem given by 159 Eqn. (4). To ensure our approximation is stable, we have instead used an empirical approach: we simulate 160 an additional cycle using the HRM and the LRMs and determine $M_{R}$ by finding $M$ that minimizes the 161 absolute error between $\mathbf{f}$ and $\mathbf{f}^{\mathrm{ROM}}$ within that cycle.

\subsection{Quantifying and Estimating Error}

164 To measure the accuracy of $\mathbf{f}^{\mathrm{ROM}}$ relative to $\mathbf{f}$, we define the averaged root mean square error (RMSE) of 165 the ROM approximation over a cycle as $\epsilon_{\mathrm{RMSE}}$ :

$$
\epsilon_{\mathrm{RMSE}}=\frac{1}{14} \sum_{i=1}^{14} e_{\mathrm{RMSE}}\left(\mathbf{f}_{i}^{\mathrm{ROM}}, \mathbf{f}_{i}\right)
$$

167 where $\mathbf{f}_{i}^{\mathrm{ROM}}$ is the ROM approximation of $\mathbf{f}_{i}, e_{\mathrm{RMSE}}\left(\mathbf{f}^{\mathrm{ROM}}, \mathbf{f}\right)$ is the RMSE between $\mathbf{f}^{\mathrm{ROM}}$ and $\mathbf{f}$, and

$168 \epsilon_{\mathrm{RMSE}}$ is averaged over the two-week cycle. Similarly, we define the PODMM approximation on the 169 coarse grid, given by $\mathbf{g}^{\mathrm{ROM}}=\overline{\mathbf{g}}+\sum_{i=1}^{M} \alpha_{i}(\mathbf{g}) \zeta_{i}^{\mathbf{g}}$, and determine its RMSE error, denoted as $e_{\mathrm{RMSE}}\left(\mathbf{g}^{\mathrm{ROM}}, \mathbf{g}_{i}\right)$, 
$171 \quad e_{\mathrm{RMSE}}\left(\mathbf{g}^{\mathrm{ROM}}, \mathbf{g}_{i}\right)$ :

$$
e_{\mathrm{EST}}(\mathbf{g})=C_{0} e_{\mathrm{RMSE}}\left(\mathbf{g}^{\mathrm{ROM}}, \mathbf{g}\right),
$$

173 where $C_{0}$ is a positive constant; $C_{0}$ is typically greater than 1 because Eqn. (4) minimizes the $e_{\mathrm{RMSE}}$ of $174 \mathbf{g}^{\mathrm{ROM}}$, and thus the $e_{\mathrm{RMSE}}$ of $\mathbf{g}^{\mathrm{ROM}}$ will be smaller than the $e_{\mathrm{RMSE}}$ of $\mathbf{f}^{\mathrm{ROM}}$. We determine $C_{0}$ by 175 computing the maximum ratio of the $e_{\mathrm{RMSE}}$ of $\mathbf{f}^{\mathrm{ROM}}$ to the $e_{\mathrm{RMSE}}$ of $\mathbf{g}^{\mathrm{ROM}}$ for the days in the additional 176 cycle used to determine $M_{R}$.

178 The error estimator $e_{\mathrm{EST}}$ serves two purposes. First, it can be used to determine time scales for which the 179 ROM will be accurate when predicting into the future. Since the $e_{\mathrm{EST}}$ is only a function of $\mathbf{g}$, it can be 180 efficiently evaluated when determining $\mathbf{f}^{\text {ROM }}$. For a desired level of accuracy, $\tau_{\text {tol }}$, we can evaluate time 181 scales in which $e_{\mathrm{EST}} \leq \tau_{\text {tol }}$; we demonstrate this in Section 2.2. Second, $e_{\mathrm{EST}}$ can also be used to determine 182 the length of the training period in a similar manner. By specifying a time scale in which we require $183 e_{\mathrm{EST}} \leq \tau_{\mathrm{tol}}$, we can adaptively increase the length of the training period until the requirement is fulfilled.

\subsection{Training and Validation Datasets}

186 The HRM described in Section 1.1 serves as the reference model in this work. Based on this compu187 tationally demanding model, we simulate a relatively short period (i.e., the initial few injection188 production cycles) to train the ROM. To distinguish between the various ROMs, we use ROM-HET, 189 ROM-HOM, and ROM-DPM to denote ROMs constructed using the LRM-HET, LRM-HOM, and LRM190 DPM, respectively. We focus on the prediction of oil saturation $\boldsymbol{S}_{\text {oil }}$ in the entire model domain and oil 191 production rate $\left(Q_{\text {oil }}\right)$, which is computed by integrating predicted $\boldsymbol{F}_{\text {oil }}$ over the boundaries of the well. We

192 examined two training periods: 5 and 15 EOR cycles (i.e., 70 and 210 days). Daily solutions from the 193 low- and high-resolution models within these periods are used to train the ROMs. Since the injection- 
194 production cycle consists of three distinct stages (injection, soaking/rest and production), separate ROMs

195 are constructed for the three stages because the solutions in each stage can have unique characteristics.

196 For example, $\boldsymbol{F}_{\text {oil }}$ is close to zero during the soaking stage, and of different characteristics during the

197 injection and production stages. We note that $M_{R}$ will be different for the three stages.

199 In the results section, we study the accuracy of the ROMs using validation sample sets consisting of the 200 daily HRM solutions determined up to the $105^{\text {th }}$ cycle (Day 1470). Depending on the training periods, the 201 validation sample set starts from the $6^{\text {th }}$ cycle (Day 71) for ROMs trained using 5 cycles, and the $16^{\text {th }}$ 202 cycle (Day 211) for ROMs trained using 15 cycles.

\section{RESULTS}

\subsection{Oil Production Rate}

206 The ROMs are able to reproduce the oil production rates $\left(Q_{\text {oil }}\right)$ very accurately (Figure 2 (a) and (b)). The 207 relative error between $Q_{\text {oil }}$ of HRM and ROM-HET, constructed using 15 training cycles, is less than 6\% 208 averaged over the production stage during the $55^{\text {th }}$ cycle. The error stays approximately the same even 209 when predicting up to the $105^{\text {th }}$ cycle. Compared to the $Q_{\text {oil }}$ obtained using LRMs, ROM-HET reduces the

210 LRM-HET's errors by $83 \%$, and $85 \%$ for the $55^{\text {th }}$, and $105^{\text {th }}$ cycles, respectively. If a lower accuracy is 211 acceptable, for example due to underlying uncertainty in the HRM, 5 training cycles may be sufficient 212 since it leads to only slight increases in the error. By performing only 6 cycles of HRM simulation instead 213 of 105 cycles based on our proposed PODMM, we can reduce the computational cost by 94\%; running 214 the LRM, and setting up and evaluating the ROM are computationally very efficient. We elaborate further 215 on the condition under which a shorter training period is possible in Section 2.3.

217 Comparing the three different ROMs (Figure 2 (a) and (b)), ROM-HET is consistently more accurate than 218 ROM-HOM and ROM-DPM. This is expected because (1) LRM-HET is more accurate than LRM-HOM 
and LRM-DPM, and (2) the variation of the approximation error with increasing $M$ has a more well-

220 defined minimum, resulting in a more accurate determination of $M_{R}$. Both reasons can be attributed to the

221 fact that LRM-HET is a better approximation of the HRM since it retains some of the heterogeneous

222 structure of the HRM.
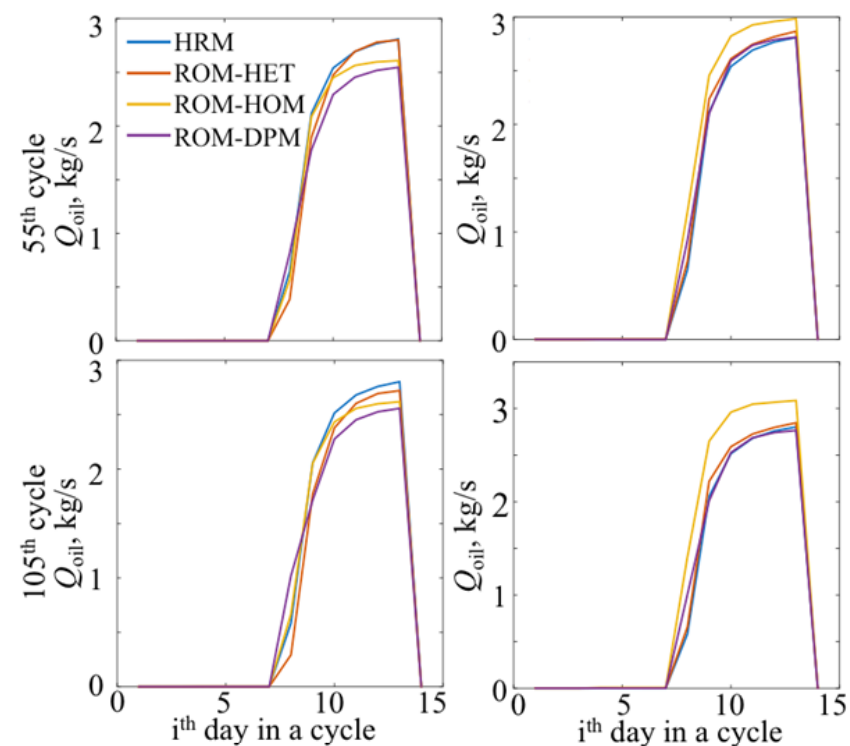

(a) ROMs, 5 train cycles
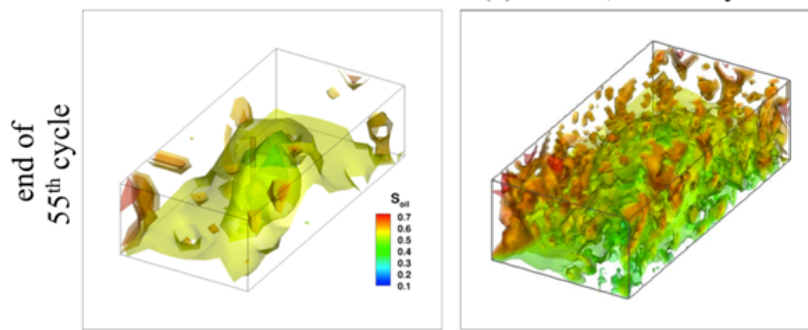

(b) ROMs, 15 train cycles

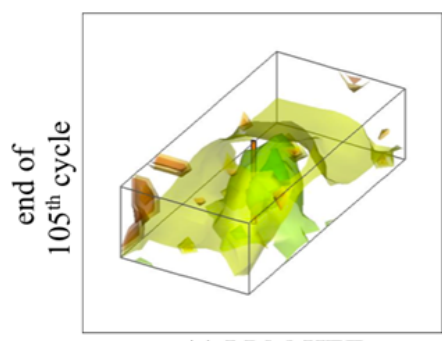

(c) LRM-HET

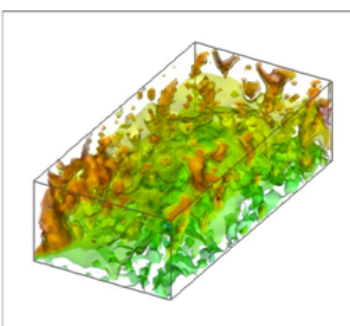

(d) HRM
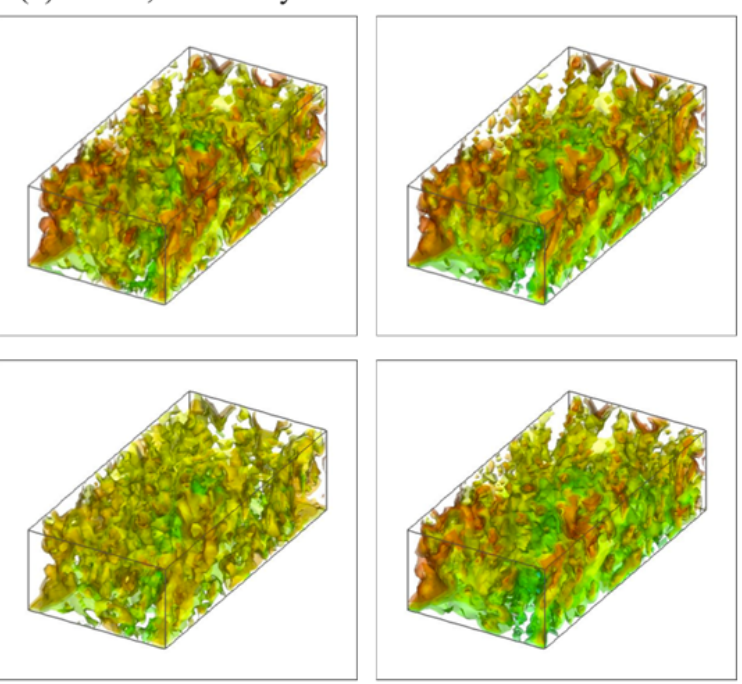

(e) ROM-HET, 5 train cycles

(f) ROM-HET, 15 train cycles

224 Figure 2. Comparison of the oil production rate $\left(Q_{\text {oil }}\right)$ obtained for the $55^{\text {th }}$ (top), and $105^{\text {th }}$ (bottom) cycles from the 3 different ROMs trained using (a) 5, and (b) 15 training cycles; and oil 

(bottom) cycles.

229 When the ROMs are trained using 15 training cycles, ROM-DPM can be as accurate as ROM-HET. 230 ROM-HOM, however, consistently over-predicts $Q_{\text {oil }}$. This over-prediction is likely due to overfitting. 231 Since the approximation error increases with time, $M_{R}$ should decrease over time to avoid overfitting. We

232 will study how $M_{R}$ should be reduced over time in the future. We note that if we have determined $M_{R}$ by 233 minimizing the absolute error of $Q_{\text {oil }}$ between the ROMs and the HRM over the entire validation period, 234 we are able to get good approximations for all ROMs. However, determining the actual error of $Q_{\text {oil }}$ over 235 the entire validation period is not practical since we want to avoid simulating the HRM over an extended 236 period of time.

\subsection{Reproduction of Fine-scale Saturation Distribution}

239 We first determine how the accuracy of the ROMs in reproducing fine-scale saturation solutions changes 240 over time. Initial tests suggest that ROM-HOM and ROM-DPM cannot reproduce the heterogeneity in the 241 solution obtained using the HRM; therefore, we will only discuss results obtained using ROM-HET in 242 this section. Figure 3(a) shows that the error, $\epsilon_{\mathrm{RMSE}}$, increases with the number of cycles after the initial 243 training period. The use of 15 instead of 5 training cycles reduces $\epsilon_{\mathrm{RMSE}}$ by $35 \%$. In addition, $\epsilon_{\mathrm{RMSE}}$ of 244 ROM-HET trained with 5 cycles grows much faster than ROM-HET trained with 15 cycles. The poorer 245 performance of ROM-HET trained with 5 cycles indicates that the initial transient dynamics of the first 5 246 cycles are poor representations of the long-time behavior of $\boldsymbol{S}_{\text {oil }}$. Subsequent slower growth of $\epsilon_{\mathrm{RMSE}}$ 247 indicates that $\boldsymbol{S}_{\text {oil }}$ changes more slowly at later times. 
248

249

250

251

252

253

254

255

256

257

258

259

260

261 We now determine how well PODMM reproduces the $\boldsymbol{S}_{\text {oil }}$ distribution. Figure 2(d) shows the

262 heterogeneous structure in $\boldsymbol{S}_{\text {oil }}$ at the end of the $55^{\text {th }}$ cycle (Day 770) and $105^{\text {th }}$ cycle (Day 1470). Figure 2

263

264

Figure 3. (a) The $\epsilon_{\mathrm{RMSE}}$ of ROM-HET increases with number of cycles after the training period. Increasing the number of training cycles from 5 to 15 reduces $\epsilon_{\mathrm{RMSE}}$. (b) For ROM-HET trained using 15 cycles, $\epsilon_{\mathrm{EST}}=\frac{1}{14} \sum_{i=1}^{14} e_{\mathrm{EST}}\left(\mathbf{g}_{i}\right)$ is close to $\epsilon_{\mathrm{RMSE}}$, indicating that $e_{\mathrm{EST}}$ is a good error estimator.

Since $\epsilon_{\mathrm{RMSE}}$ increases with time, the ROM-HET we constructed is only accurate for a finite number of cycles. To determine the range of cycles for which the ROM-HET is valid without performing HRM simulation over an extended period, we will use the error estimator $e_{\mathrm{EST}}$ defined in Eqn. (5). For the ROM-HET trained with 15 cycles, $C_{0}$, determined based on the $16^{\text {th }}$ cycle, is found to be 10.334 . Figure 3(b) shows that $\epsilon_{\mathrm{EST}}=\frac{1}{14} \sum_{i=1}^{14} e_{\mathrm{EST}}\left(\mathbf{g}_{i}\right)$, the mean of $e_{\mathrm{EST}}$ over a cycle, is a good predictor of $\epsilon_{\mathrm{RMSE}}$. We can thus determine a time scale for which the ROM-HET has desired accuracy by specifying an appropriate tolerance for $\epsilon_{\mathrm{EST}}$. For example, if we specify a tolerance of 0.05 , the ROM-HET that we have constructed has the desired accuracy up to the $74^{\text {th }}$ cycle for predicting $\boldsymbol{S}_{\text {oil }}$.

(f) shows that ROM-HET trained with 15 cycles is able to reproduce most of the intricate structure of $\boldsymbol{S}_{\text {oil }}$ on the $2 \mathrm{~m}$ scale. However, ROM-HET is not able to reproduce the very high $\boldsymbol{S}_{\mathrm{oil}}$ values; the

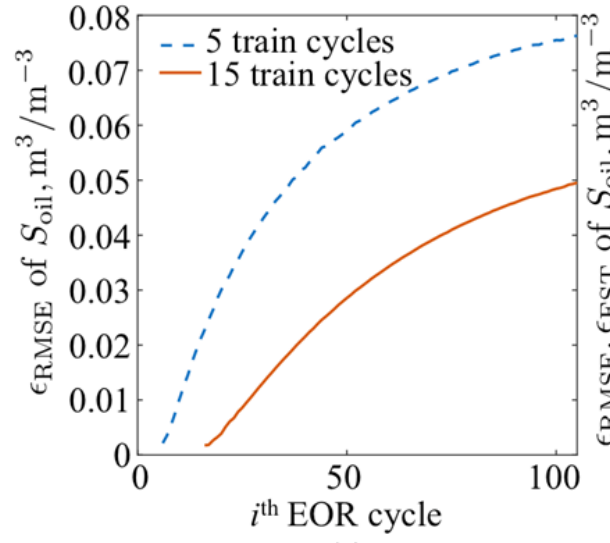

(a)

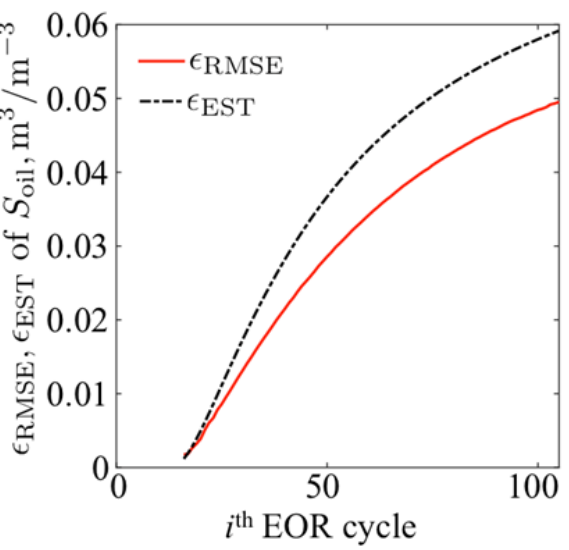

(b) 
265 approximated solutions are smoother since only 9 POD bases are included in the approximation. 266 Although the ignored POD bases amount to only $0.04 \%$ of the variance in the solutions within the 267 training period, they can have a significant impact on the approximation of the solutions at later times, 268 especially if these solutions are significantly different from the solutions in the training period. Consistent 269 with the results in Figure 3, ROM-HET trained with 5 cycles has poorer predictive capability (Figure 2 270 (e)). Nonetheless, in both cases, ROM-HET added a significant amount of small-scale structure to the 271 LRM results shown in Figure 2(c). In this respect, the PODMM can be viewed as a downscaling 272 procedure. With 15 training cycles (plus one additional cycle for determining $M_{R}$ ), the computational cost 273 of simulating 105 cycles is reduced by $85 \%$.

\subsection{Dependence of training period on fine-scale reproduction}

276 Compared to $Q_{\text {oil }}$, the estimation of $\boldsymbol{S}_{\text {oil }}$ requires a longer training period. Approximating $\boldsymbol{S}_{\text {oil }}$ over the 277 entire domain requires reproducing a significant amount of fine-scale information and is thus a 278 challenging problem. On the other hand, $Q_{\text {oil }}$ is a scalar, integrated quantity over a smaller region of the 279 domain near the well and does not need to accurately capture fine-scale information in the $\boldsymbol{F}_{\text {oil }}$ over the

280 entire domain. We can thus obtain sufficiently accurate prediction even with a shorter training period 281 (Figure 2(a)). For the current problem where the goal is to predict future solutions, we can conclude that a 282 longer training period is needed to accurately capture fine-scale features in a solution. If we are interested 283 in capturing the effects of operational parameters (e.g., injection rate, injection duration, production rate 284 and production duration) and model parameters (e.g., rock properties), the training period will 285 additionally depend on how the $\boldsymbol{S}_{\text {oil }}$ and $Q_{\text {oil }}$ vary with these parameters within the modeled parameter 286 space. 


\section{CONCLUSIONS AND FUTURE WORK}

289 We have demonstrated that PODMM is capable of predicting long-time behavior of the oil production 290 rate $\left(Q_{\text {oil }}\right)$ and oil saturation $\left(\boldsymbol{S}_{\text {oil }}\right)$. The main conclusions are: models (e.g., subsurface flow coupled to geomechanics) to study and improve the robustness of PODMM.

\section{ACKNOWLEDGMENT}

307 We would like to thank the three anonymous reviewers for their constructive comments. This research 308 was supported, in part, by the U.S. Department of Energy under Contract \#DE-AC02-05CH11231. We 309 thank Rishi Parashar of the Desert Research Institute for making ThreeDFracMap available to this 310 project. 
312

313

314

315

316

317

318

319

320

321

322

323

324

325

326

327

328

329

330

331

332

333

334

335

336

337

338

339

340

341

342

343

344

345

346

347

348

349

350

351

352

353

354

355

356

357

358

359

360

361

[1] Robinson T, M Eldred, K Willcox, R Haimes. Strategies for Multifidelity Optimization with Variable Dimensional Hierarchical Models. 47th AIAA/ASME/ASCE/AHS/ASC Structures, Structural Dynamics, and Materials Conference. American Institute of Aeronautics and Astronautics, Newport, Rhode Island, 2006.

[2] Pau GSH, G Bisht, WJ Riley. A reduced-order modeling approach to represent subgrid-scale hydrological dynamics for land-surface simulations: application in a polygonal tundra landscape. Geosci Model Dev. 7 (2014) 2091-105, doi: 10.5194/gmd-7-2091-2014.

[3] Pau GSH, C Shen, WJ Riley. Accurate and efficient prediction of fine-resolution hydrologic and carbon dynamic simulations from coarse-resolution models. (2015). Submitted.

[4] Wilby RL, TML Wigley, D Conway, PD Jones, BC Hewitson, J Main, et al. Statistical downscaling of general circulation model output: A comparison of methods. Water Resour Res. 34 (1998) 2995-3008, doi: 10.1029/98WR02577.

[5] Fowler HJ, S Blenkinsop, C Tebaldi. Linking climate change modelling to impacts studies: recent advances in downscaling techniques for hydrological modelling. International Journal of Climatology. 27 (2007) 1547-78, doi: 10.1002/joc.1556.

[6] Gutmann E, T Pruitt, MP Clark, L Brekke, JR Arnold, DA Raff, et al. An intercomparison of statistical downscaling methods used for water resource assessments in the United States. Water Resour Res. 50 (2014) 7167-86, doi: 10.1002/2014WR015559.

[7] von Storch H, E Zorita, U Cubasch. Downscaling of Global Climate Change Estimates to Regional Scales: An Application to Iberian Rainfall in Wintertime. Journal of Climate. 6 (1993) 1161-71.

[8] Hanssen-Bauer I, EJ Forland, JE Haugen, OE Tveito. Temperature and precipitation scenarios for Norway: comparison of results from dynamical and empirical downscaling. Climate Research. 25 (2003) 15-27, doi: DOI 10.3354/cr025015.

[9] Higdon D, J Gattiker, B Williams, M Rightley. Computer model calibration using high-dimensional output. Journal of the American Statistical Association. 103 (2008) 570-83.

[10] Wilkinson RD. Bayesian calibration of expensive multivariate computer experiments. Large-Scale Inverse Problems and Quantification of Uncertainty. 707 (2011) 195-215.

[11] Everson R, L Sirovich. Karhunen-Loeve procedure for gappy data. Journal of the Optical Society of America A. 12 (1995) 1657-64.

[12] Winton C, J Pettway, CT Kelley, S Howington, OJ Eslinger. Application of Proper Orthogonal Decomposition (POD) to Inverse Problems in Saturated Groundwater Flow. Adv Water Resour. (2011) 1-24, doi: 10.1016/j.advwatres.2011.09.007.

[13] Boyce SE, T Nishikawa, WWG Yeh. Reduced order modeling of the Newton formulation of MODFLOW to solve unconfined groundwater flow. Adv Water Resour. 83 (2015) 250-62, doi: 10.1016/j.advwatres.2015.06.005.

[14] Li H, Z Luo, J Chen. Numerical simulation based on POD for two-dimensional solute transport problems. Applied Mathematical Modelling. 35 (2011) 2489-98, doi: 10.1016/j.apm.2010.11.064.

[15] Siade AJ, M Putti, WWG Yeh. Snapshot selection for groundwater model reduction using proper orthogonal decomposition. Water Resour Res. 46 (2010) W08539, doi: 10.1029/2009WR008792.

[16] Kunisch K, S Volkwein. Galerkin Proper Orthogonal Decomposition Methods for a General Equation in Fluid Dynamics. SIAM Journal on Numerical Analysis. 40 (2002) 492-515, doi: $10.1137 /$ S0036142900382612.

[17] Luo Z, J Du, Z Xie, Y Guo. A reduced stabilized mixed finite element formulation based on proper orthogonal decomposition for the non-stationary Navier-Stokes equations. International Journal for Numerical Methods in Engineering. 88 (2011) 31-46, doi: 10.1002/nme.3161.

[18] Willcox K, J Peraire. Balanced model reduction via the proper orthogonal decomposition. AIAA journal. 40 (2002) 2323-30.

[19] Barrault M, Y Maday, NC Nguyen, AT Patera. An 'empirical interpolation' method: application to efficient reduced-basis discretization of partial differential equations. Comptes Rendus Mathematique. 339 (2004) 667-72, doi: 10.1016/j.crma.2004.08.006. 
362 [20] Constantine PG, Q Wang. Residual minimizing model interpolation for parameterized nonlinear 363 dynamical systems. SIAM Journal on Scientific Computing. 34 (2012) A2118-A44, doi: $36410.1137 / 100816717$.

365 [21] Chaturantabut S, D Sorensen, J Steven. Nonlinear model reduction via discrete empirical interpolation. SIAM Journal on Scientific Computing. 32 (2010) 2737-64.

367 [22] Parashar R, DM Reeves. Computation of flow and transport in fracture networks on a continuum 368 grid. Proceedings of MODFLOW and More: Integrated Hydrologic Modeling, Golden, CO, 2011.

369 [23] Pruess K, G Moridis, C Oldenburg. TOUGH2 User's Guide, Version 2.1, Report LBNL-43134. 370 Lawrence Berkeley National Laboratory, Berkeley, California 1999.

371 [24] Pruess K, TN Narasimhan. A Practical Method for Modeling Fluid and Heat-Flow in Fractured 372 Porous-Media. Society of Petroleum Engineers Journal. 25 (1985) 14-26, doi: 10.2118/10509-PA. 